\title{
Research on Pattern Recognition Method of Blockage Signal in Pipeline Based on LMD Information Entropy and ELM
}

\author{
Jingzong Yang, ${ }^{1,2}$ Xiaodong Wang, ${ }^{1,2}$ Zao Feng, ${ }^{1,2}$ and Guoyong Huang ${ }^{1,2}$ \\ ${ }^{1}$ Faculty of Information Engineering and Automation, Kunming University of Science and Technology, Kunming 650500, China \\ ${ }^{2}$ Engineering Research Center for Mineral Pipeline Transportation of Yunnan Province, Kunming 650500, China \\ Correspondence should be addressed to Xiaodong Wang; wxdedu@foxmail.com
}

Received 17 December 2016; Revised 30 January 2017; Accepted 7 February 2017; Published 6 March 2017

Academic Editor: Yuri Vladimirovich Mikhlin

Copyright (c) 2017 Jingzong Yang et al. This is an open access article distributed under the Creative Commons Attribution License, which permits unrestricted use, distribution, and reproduction in any medium, provided the original work is properly cited.

\begin{abstract}
Aiming at the nonstationary and nonlinear characteristics of acoustic impulse response signal in pipeline blockage and the difficulty in identifying the different degrees of blockage, this paper proposed a pattern recognition method based on local mean decomposition (LMD), information entropy theory, and extreme learning machine (ELM). Firstly, the impulse response signals of pipeline extracted in different operating conditions were decomposed with LMD method into a series of product functions (PFs). Secondly, based on the information entropy theory, the appropriate energy entropy, singular spectrum entropy, power spectrum entropy, and Hilbert spectrum entropy were extracted as the input feature vectors. Finally, ELM was introduced for classification of pipeline blockage. Through the analysis of acoustic impulse response signal collected under the condition of health and different degrees of blockages in pipeline, the results show that the proposed method can well characterize the state information. Also, it has a great advantage in terms of accuracy and it is time consuming when compared with the support vector machine (SVM) and BP (backpropagation) model.
\end{abstract}

\section{Introduction}

Pipeline transportation is one of the world's fifth largest types of transportation, which has been widely used in metallurgy, coal, hydropower, and other departments of material transportation. Compared with railway, highway, water, and air transport, pipeline transportation has many advantages, such as energy saving, environmental protection, and high efficiency. However, the safety problem of pipeline transportation is becoming more prominent, especially in long distance water delivery pipeline, which plays an important role in the urban infrastructure. Because the unreasonable design of pipeline, long time operation, or low flow rate of fluid, the phenomenon of sediment deposition in the pipeline is very common. Most of the blockage accidents in the early days showed different degrees of partial blockage, and it is easy to be ignored by the inspector. This phenomenon causes a gradual expansion of the blocked area and eventually leads to the abnormal operation of pipeline. The accident of blockage not only greatly increases the pipeline operating costs but also causes the decrease of transport capacity. So, the judgment of pipeline operation in a timely manner is necessary and can prevent the irreparable loss caused by these accidents.

The methods used for pipeline blockage detection usually including percussion sound method, pipeline strain method, hydraulic analysis method, mass-volume balance method, and acoustic detection method, through analyzing the acoustic signal which is based on acoustic detection method, have a lot of advantage [1], such as high sensitivity with no damage in pipeline equipment and high detecting speed. Thus it has been widely used in actual detection [2-4]. However, the collected acoustic impulse response signal is nonstationary and nonlinear, and single time domain or frequency domain analysis cannot be used for pattern recognition effectively. General time-frequency analysis methods include wavelet or wavelet packet decomposition, empirical mode decomposition (EMD), ensemble empirical mode decomposition (EEMD), and local mean decomposition (LMD). LMD is a new adaptive method of nonstationary signal analysis, which 
was first put forward by Smith in 2005 [5] and has been applied to electroencephalograph (EEG) spectrum analysis. Compared with short-time Fourier transform (STFT), wavelet transform, and other methods, LMD has a strong adaptability. Meanwhile, the performance of iterative efficiency and restraining the border effects are better than EMD method [6]. Also, LMD can decompose a complex nonstationary signal into a number of PF components and each PF component is obtained by multiplication of the instantaneous amplitude and pure frequency modulation signal. So, it also has a better effect in maintaining the signal frequency and envelope information. It is a demodulation process to decompose the acoustic impulse response signal of pipeline through LMD method under different operating conditions, which provide a strong foundation for the further extraction of signal characteristics. Thus this method is suitable for analysis and diagnosis of acoustic impulse response signals in pipeline blockage.

Information entropy, as a well-known information processing technology, relates the amount of uncertainty about an event associated with a given probability distribution. It is mainly used for data compression and coding. At present, it has been applied in the field of fault diagnosis and achieved remarkable results. Through the analysis of EMD energy entropy of different vibration signals, Yu et al. [7] recognized the different failure modes of the bearing effectively. Yan and Gao [8] proposed a method of machine health monitoring based on approximate entropy, and the experiments show that the approximate entropy can effectively characterize the safety structure defect. Through the analysis of the start transient with current information entropy theory, Cabal-Yepez et al. [9] quantitatively inspected different fault induction motor signals and thus identified the motor's health state.

As a kind of artificial intelligence method, neural network has been widely used in the field of fault diagnosis [10-12]. With the ability of self-organization and self-learning, the network itself can achieve good classification. However, it is easy to fall into overfitting or local optimum, and it also requires a lot of data samples in its practical application. Extreme learning machine (ELM) [13] that was developed in recent years is simple in structure and very convenient to use. Meanwhile, except for solving the problem of local optimum generated by the neural network, ELM only need to set the number of hidden layer nodes, the input weights, and the bias of the hidden elements to obtain the global optimal solution. So, it has been widely used and developed in the field of pattern recognition [14-16].

Based on this, LMD, information entropy, and ELM algorithm are combined to identify and analyze the acoustic impulse response signal of blockage in pipeline. Firstly, through the signal pretreatment of LMD and EMD, the experiments obtain a series of PF components and Intrinsic Mode Function (IMF) components. Secondly, according to the theory of information entropy, the appropriate energy entropy, singular spectrum entropy, power spectrum entropy, and Hilbert spectrum entropy are extracted as the feature vectors and then input to ELM classifier to obtain the classification results. Finally, ELM is compared to support vector machine (SVM) and BP neural network model which are more popular for pattern recognition currently.

This paper is organized as follows. Section 2 not only introduces LMD algorithm, ELM algorithm, and the principle of sound propagation in pipeline but also analyzes the experimental principle based on the acoustic detection. In Section 3, the feature extraction method based on LMD information entropy is proposed. Section 4 presents the pattern recognition model based on LMD information entropy and ELM. The actual experimental analysis is presented in Section 5. Conclusions are obtained in Section 6.

\section{Basic Principle}

2.1. Sound Propagation in Pipeline. The equation of one dimensional acoustic wave is derived from the kinetic equation, continuity equation, and state equation [17-19]. The equation of three-dimensional acoustic wave is as follows:

$$
\nabla^{2} p=\frac{1}{c_{o}^{2}} \frac{\partial^{2} p}{\partial t^{2}}
$$

where $c_{o}$ is the propagation speed of acoustic wave. $p$ is the sound pressure. $t$ is the transmission time. $\nabla^{2}$ is the Laplacian and it can be described by the Cartesian coordinates as follows:

$$
\nabla^{2}=\operatorname{div}(\operatorname{grad} p)=\frac{\partial^{2}}{\partial x^{2}}+\frac{\partial^{2}}{\partial y^{2}}+\frac{\partial^{2}}{\partial z^{2}}
$$

According to the different wave front, acoustic wave can be divided into three types: the spherical wave, the cylindrical wave, and the plane wave. The plane wave refers to the acoustic wave which propagates only one direction, and all the particles in other directions have the same amplitude and phase. The sound pressure of plane wave is as follows:

$$
p=A e^{j(\omega t-k x)}
$$

where $A$ is the pressure amplitude of incident wave, $k(k=$ $\omega / c)$ is the wave number of acoustics, $c$ is the speed of sound, $\omega(\omega=2 \pi f)$ is angular frequency, $f$ is the frequency of acoustic wave, and $x$ is the propagation distance. So formula (1) in the cylindrical coordinates can be expressed as follows:

$$
\frac{1}{r} \frac{\partial}{\partial r}\left(r \frac{\partial p}{\partial r}\right)+\frac{1}{r^{2}} \frac{\partial^{2} p}{\partial \theta^{2}}+\frac{\partial^{2} p}{\partial z^{2}}=\frac{1}{c_{o}^{2}} \frac{\partial^{2} p}{\partial t^{2}} .
$$

The cylindrical coordinates of pipeline are shown in Figure 1 [20]. Formula (4) not only reflects the change of sound pressure along with the change of time $(t)$ and space position $(x, y, z)$ but also shows a common rule of wave phenomena. When the acoustic wave propagates in the actual medium, its intensity can decrease with the increase of propagation distance. Meanwhile, a part of the acoustic energy is consumed to form heat energy. This phenomenon is called acoustic attenuation. The attenuation of acoustic wave mainly includes three kinds of forms: absorption attenuation, scattering attenuation, and diffused attenuation.

When the acoustic wave propagates in the pipeline, the reflection and refraction can be generated at the interface of the tube wall, which can influence the propagation of the acoustic wave. As shown in Figure 2 [20], only the longitudinal wave can be spread in the fluid medium I. The incident wave $p_{i L}$ and reflection wave $p_{r L}$ are all longitudinal waves. The speed of sound is $c_{1 L}$. Meanwhile, there exist refracted longitudinal wave $p_{t L}$ and refraction wave $p_{t T}$ in the 


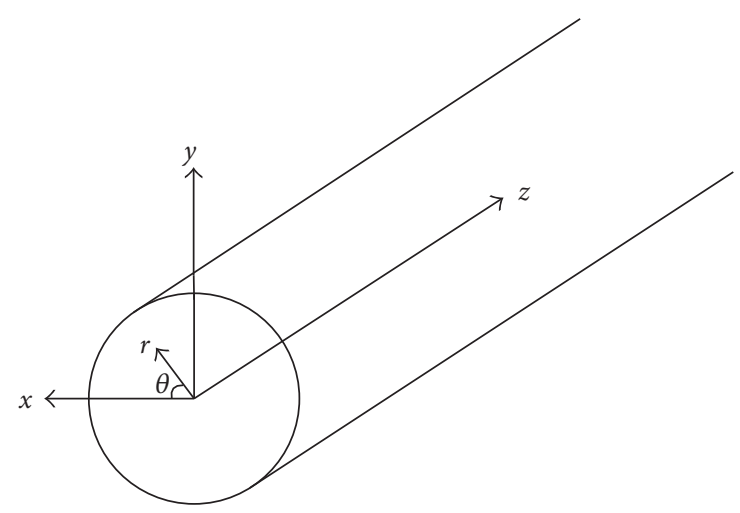

FIgURe 1: Cylindrical coordinates.

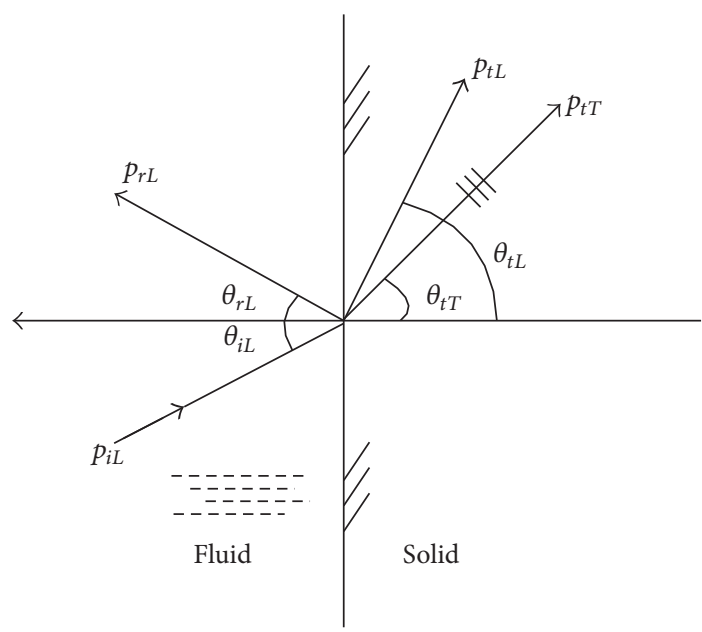

FIGURE 2: Reflection and transmission of oblique incident wave through the interface of liquid-solid medium.

solid medium II. Due to the boundary condition of the solidliquid interface, the normal component of the sound pressure at both sides of the interface is continuous. The emergence angle $\theta_{i}$, reflection angle $\theta_{r}$, and refraction angle $\theta_{t}$ can be derived as follows:

$$
\begin{aligned}
\theta_{i} & =\theta_{r}, \\
\frac{\sin \theta_{i}}{\sin \theta_{t}} & =\frac{c_{1}}{c_{2}} .
\end{aligned}
$$

Because the reflection and refraction of acoustic wave that are located at the plane interface can meet the law of Snell [21] and the acoustic speed of longitudinal wave $c_{2 L}$ in the steel tube is greater than that of transverse wave $c_{2 T}$, the refraction angle of refracted longitudinal wave $\theta_{t L}$ is always greater than the angle of refraction transverse wave $\theta_{t T}$.

By studying the boundary conditions of plane wave and the expressions which are introduced from formula (1) to formula (5), the following relationship can be derived:

$$
\frac{\sin \theta_{r L}}{c_{1 L}}=\frac{\sin \theta_{i L}}{c_{1 L}}=\frac{\sin \theta_{t L}}{c_{2 L}}=\frac{\sin \theta_{t T}}{c_{2 T}} .
$$

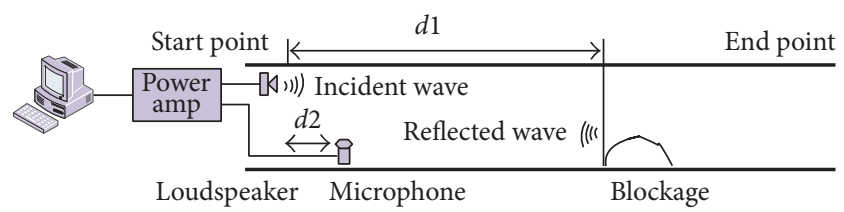

FigURE 3: Schematic diagram of principle in pipeline blockage detection.

When the above acoustic speed meets $c_{2 L}>c_{2 T}>c_{1 L}$, there are two critical angles of total internal reflection angle in the medium I; namely,

$$
\begin{gathered}
\text { First critical angle: } \theta_{1}=\arcsin \frac{c_{1 L}}{c_{2 L}}, \\
\text { Second critical angle: } \theta_{2}=\arcsin \frac{c_{1 L}}{c_{2 T}} .
\end{gathered}
$$

When the condition of $\theta_{i}<\theta_{r}<\theta_{1}$ is satisfied, there exist both refraction longitudinal wave and refraction transverse wave in the solid medium I. Meanwhile, with the increase of the angle, the refraction wave is gradually weakened, and the shear wave is gradually strengthened. When the condition of $\theta_{1}<\theta_{i}<\theta_{2}$ is satisfied, longitudinal wave can be all reflected, and there exists only transverse wave in the solid medium. When the condition of $\theta_{2} \leq \theta_{i}$ is satisfied, there exists only internal reference in the solid medium $\prod$.

2.2. The Experimental Principle and the Extraction of Impulse Response Signal. The experiment is carried out in a pipeline with a diameter much smaller than the wavelength of acoustic wave. When the diameter of pipeline is smaller than the wavelength of acoustic wave, the acoustic wave can spread in the pipeline in the form of a plane wave. Moreover, the wave front remains with the same size, so the sound pressure amplitude does not vary with the change of distance. This shows that the energy of acoustic wave does not decay during the propagation along a pipeline. Therefore, only the reflection and refraction of sound waves need to be considered under this kind of condition.

The detection theory of pipeline blockage and systematic block diagram of pipeline blockage detection are shown in Figures 3 and 4, respectively. The sensor is installed at one end of the pipeline and another end was open. The distance between loudspeaker and blocking object is $d 1$. The distance between microphone and loudspeaker is $d 2$. The experiment applied an impulse signal to the pipeline through an amplifier driver. When acoustic wave is encountered by the obstacles, part of the acoustic wave can be reflected back, and the microphone is used to record the impulse signal received by the whole process. Therefore, the specific information of blockage can be obtained by further analysis of the response signals. In addition, the acoustic signals collected in the experiment are all sound pressure signals.

One of the main goals of data preprocessing is to deal with the measured data obtained from the data acquisition, which can make the data more suitable for the further processing in feature extraction and classification. At present, many acoustic detection methods are based on impulse response. 


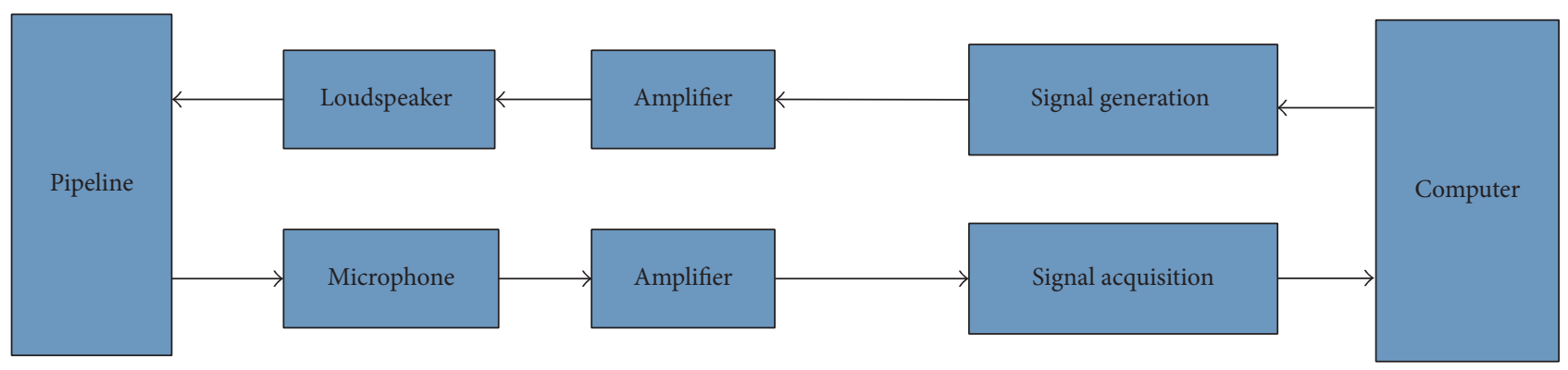

FIGURE 4: Systematic block diagram of pipeline blockage detection.

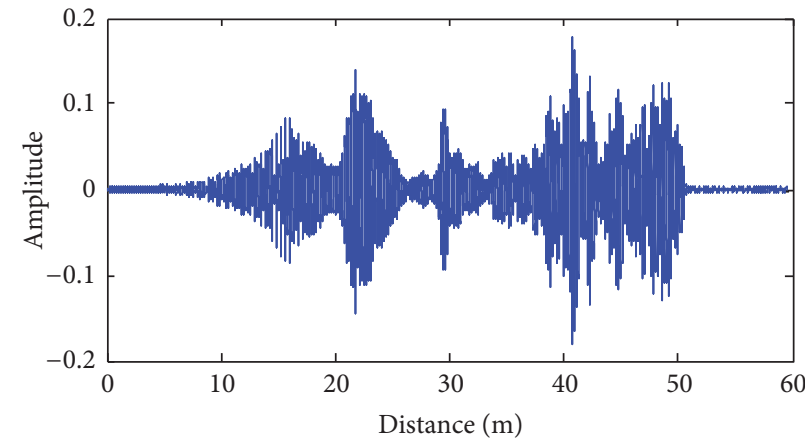

(a)

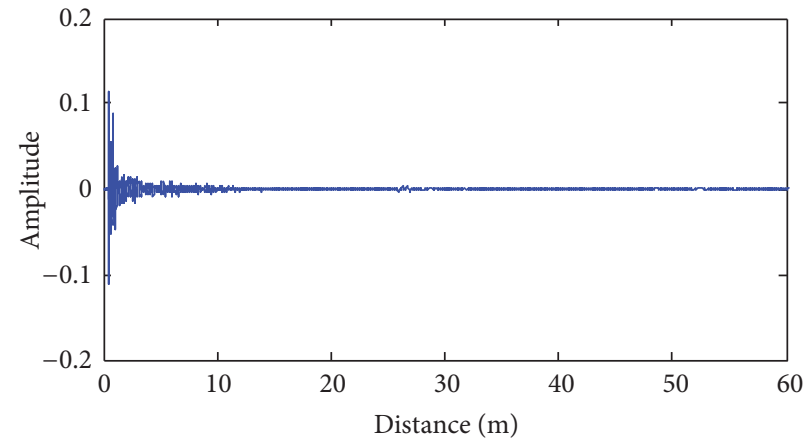

(b)

FIGURE 5: Recorded acoustic signal on empty pipeline (a) and its impulse response (b).

The application of this method is originally developed as a seismological technique for the observation of stratifications in the earth's crust [22]. When an impulsive pressure wave is produced by a source, reflections occur due to impedance differences between the layers in the earth crust. These reflections return to the surface where they are recorded. The impulsive nature of the excitation the recorded signal is termed the input impulse response. Through the analysis of the impulse response signal, the scientist can get the useful information of the earth's crust.

Today, the acoustic detection method based on impulse response has been preliminarily applied in industries, such as the quality control of pipeline in chemical engineering, oil, and natural gas. Also, acoustic impulse response of pipeline is a very useful physical quantity [23], which has been applied to the detection of defects in the pipeline (blocking, leakage) [24]. Since the impulse response contains detailed information on the geometry of the system, the sound speed, and its boundary conditions, any property changes can be reflected in acoustic impulse response. In these experiments, the impulse response signals are obtained through deconvolution of the original signals by using the convolution theorem [25]:

$$
h(t)=\operatorname{Re}\left\{F^{-1}\left[F\left\{\frac{y(t)}{(F\{x(t)\}+\gamma)}\right\}\right]\right\}
$$

where $x(t)$ is the excitation signal, $y(t)$ is the signal recorded on microphone, and $\gamma$ is the regularization factor. Figure 5 shows a recorded acoustic signal of an empty pipeline and the corresponding impulse response.

2.3. LMD Algorithm. The core idea of the local mean decomposition (LMD) method is to decompose an original signal into a number of product functions (PF), and each PF component is obtained by multiplying an envelope signal and a pure frequency modulation signal. This shows that it can obtain pure frequency modulation (FM) information and envelope information of the signal. By analyzing the instantaneous amplitude and instantaneous frequency of all PF components obtained from the decomposition, the complete time-frequency distribution of the original signal can be obtained. So, the method can get effective information that reflect the working state or fault characteristics by extracting feature vector from the signal. For any original signal $x(t)$, each PF component decomposed by LMD can be expressed as follows [26]:

$$
\mathrm{PF}_{i}(t)=a_{i}(t) s_{i}(t),
$$

where $a_{i}(t)$ is the instantaneous amplitude of PF components. Instantaneous frequency $f(x)$ can be obtained through the pure FM signal $s_{i}(t)$ :

$$
f(x)=\frac{1}{2 \pi} \frac{d\left[\arccos \left(s_{i}(t)\right)\right]}{d t} .
$$

By separating $\mathrm{PF}_{1}(t)$ from the signal $x(t)$, the signal $u_{1}(t)$ can be obtained, and then $u_{1}(t)$ is regarded as the original 


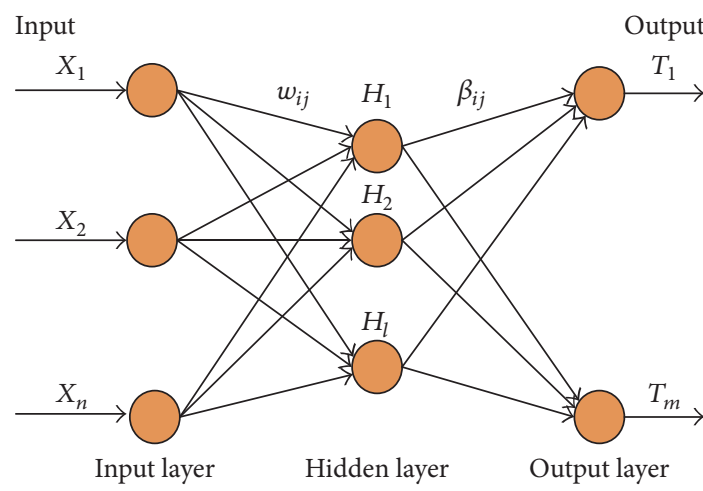

FIgURE 6: Topology diagram of ELM.

signal and cycle of $K$ times is repeated, until $u_{k}$ is a monotone function.

$$
\begin{gathered}
u_{1}(t)=x(t)-\mathrm{PF}_{1}(t) \\
u_{2}(t)=u_{1}(t)-\mathrm{PF}_{2}(t) \\
\vdots \\
u_{k}(t)=u_{k-1}(t)-\mathrm{PF}_{k}(t),
\end{gathered}
$$

where $a_{i}(t)$ and $f_{i}(t)$ represent the instantaneous amplitude and instantaneous frequency, respectively, and $N$ is the number of PF component. The original $x(t)$ is decomposed into $k \mathrm{PF}$ components and sum of a monotone function $u_{k}(t)$; namely,

$$
x(t)=\sum_{p=1}^{k} \mathrm{PF}_{p}(t)+u_{k}(t) .
$$

2.4. ELM Algorithm. Extreme learning machine (ELM) is developed on the basis of single-hidden-layer feedforward networks (SLFN), and SLFN is a special case of BP network with one layer of hidden layer. The architecture of an ELM can be described in Figure 6 [14].

Assume that the input vector and the output vector of network are $\left\{x_{i} \mid i=1,2, \ldots, n\right\}$ and $\left\{T_{j} \mid j=1,2, \ldots\right.$, $m\}$, respectively. Moreover, the hidden layer is $l$, and the activation function of the hidden layer neurons is $g(x)$. Then the whole output of the network $T$ can be expressed as follows [27]:

$$
\begin{aligned}
T & =\left[t_{1}, t_{2}, \ldots, t_{j}\right]_{n \times m}=\left[\sum_{i=1, j=1}^{l, m} \beta_{i j} g\left(w_{i j} x_{i}+b_{i}\right)\right]_{n \times m} \\
& =\left(H_{m \times l} \beta_{l \times n}\right) .
\end{aligned}
$$

$H$ denotes the output matrix of hidden layer. If the activation function $g(x)$ is infinite differentiable, SLFN can be converted to ELM. At this time, the connection weights $w$ and the hidden layer threshold $b$ can be randomly selected before training, and they can remain unchanged at the training process. This shows that it can greatly reduce the amount of computation. Furthermore, the connection weight $\beta$ can be obtained by solving the following formula:

$$
\min _{\beta}\left\|H \beta-T^{\prime}\right\| \text {. }
$$

Its solution is $\widehat{\beta}\left(\widehat{\beta}=H^{+} T^{\prime}\right)$. $T^{\prime}$ is the rank of matrix $T$. $H+$ is the Moore-Penrose generalized inverse of the hidden layer output matrix $H$. Therefore, the model-building step of ELM can be summarized as follows.

Step 1. The weight $w$ and threshold of hidden layer neurons $b$ are randomly given.

Step 2. According to BP network method to determine the number of hidden layer neurons of $\operatorname{ELM}(l=2 \times n+1)$, where $l$ is the number of hidden layer neurons, $n$ is the number of input layer neurons.

Step 3. Choose an activation function $g(x)$ which is infinite differentiability.

Step 4. Calculate the output matrix of hidden layer $H$.

Step 5. Calculate the output weight $\widehat{\beta}$.

\section{Feature Parameter Extraction Based on LMD Information Entropy}

The theory of information entropy was first put forward by scholars Shannnon [28]. According to his theory, there is a direct correlation between the degree of disorder and the values in information entropy: the larger the value of information entropy, the higher the degree of disorder in information which it indicates. So this kind of theory can well describe the uncertainty of the average probability of system. If the blocking state of pipeline changes, the frequency components of different frequency band and energy distribution of signal spectrum can follow this change. Also, it can be measured by the information entropy. Therefore, on the basis of LMD decomposition, this paper introduces the concept of information entropy and further applies it to the feature extraction. The calculation formula of information entropy is as follows [29]:

$$
G(x)=-\sum_{i=1}^{n} P\left(x_{i}\right) \log P\left(x_{i}\right),
$$

where $G(x)$ is the entropy, $P\left(x_{i}\right)$ represents the probability that an event occurs in a system, and $n$ represents the number of samples to be analyzed.

Because the acoustic impulse response signal of pipeline blockage is a complex nonstationary and nonlinear signal, a single time domain and frequency domain analysis can only describe the signal from the global perspective. It cannot make local analysis. In order to fully reflect the blocking state of the pipeline, this paper intends to extract the characteristics of signal in time domain (energy entropy and singular spectrum entropy), frequency domain (power spectral entropy), and time-frequency domain (Hilbert spectrum entropy) for the recognition of the blocking state. 
3.1. Extraction of Singular Spectrum Entropy Based on LMD. Singular value decomposition (SVD) is a kind of special matrix transformation, which can be used to characterize the essential character of signal. So it has been widely used in signal processing and data compression in recent years. Assume that the measurement data is used to construct $m \times n$ order matrix $H$ and then the singular value decomposition of $H$ is as follows [30]:

$$
H=U \Sigma V^{T}=\sum_{i=1}^{r} \sigma_{i} u_{i} v_{i}^{T}=\sum_{i=1}^{r} \sigma_{i} A_{i},
$$

where $U \in R^{m \times m}$ and $V \in R^{n \times n}$ are orthogonal matrices, $\Sigma=$ $\left[\begin{array}{cc}\Sigma_{r} & O \\ O & O\end{array}\right] \in R^{m \times n}, \Sigma_{r}=\operatorname{diag}\left(\sigma_{1}, \sigma_{2}, \ldots, \sigma_{r}\right), \sigma_{i}(i=1,2, \ldots, r)$ is called singular value of $H$, and $\sigma_{1} \geq \cdots \sigma_{r} \geq 0, r(r \leq$ $\min (m, n))$ is the rank of $H . \sigma_{1}, \ldots, \sigma_{r}$ are called the singular value of matrix $A$.

Because the different blocking state of pipeline acoustic impulse response signal can change in frequency distribution, singular value of the PF component matrix which obtained by LMD method can also appear to be changed. In order to quantitatively show the extent of this change, the singular spectrum entropy is extracted as a feature vector. The calculation steps are as follows:

(1) The collected acoustic impulse response signal is decomposed with LMD method.

(2) Get the feature vector of singular value $\lambda_{1} \geq \lambda_{2} \cdots \geq$ $\lambda_{n}$ by SVD, and then each PF component is normalized:

$$
Q_{i}=\sum_{i=1}^{n} \frac{\lambda_{i}}{Q}
$$

where $Q=Q_{1}+Q_{2} \cdots+Q_{n}, Q_{i}=\lambda_{i}$, and $\sum_{i=1}^{n} Q_{i}=1$.

(3) Calculate the singular spectrum entropy:

$$
S=-\sum_{i=1}^{n} P\left(x_{i}\right) \log P\left(x_{i}\right), \quad P\left(x_{i}\right)=\frac{Q_{i}}{Q} .
$$

3.2. Extraction of Energy Entropy Based on LMD. Because different blocking states of the acoustic impulse response signal in pipeline are different in frequency distribution, there is a certain difference in energy distribution. Based on this, the energy entropy is used as a feature vector to be extracted. The calculation steps are as follows:

(1) The collected acoustic impulse response signal is decomposed with LMD method. Then, compute their energy $E_{i}$ :

$$
E_{i}(x)=\int_{-\infty}^{+\infty}\left|\mathrm{PF}_{i}\right|^{2} d t
$$

(2) The energy of all PF components is normalized:

$$
\begin{aligned}
P\left(x_{i}\right) & =\frac{E_{i}}{E}, \\
E & =\sum_{i=1}^{n} E_{i} .
\end{aligned}
$$

(3) Calculate the energy entropy:

$$
H(x)=-\sum_{i=1}^{n} P\left(x_{i}\right) \log P\left(x_{i}\right) .
$$

3.3. Extraction of Power Spectrum Entropy Based on LMD. Although energy entropy can represent the variation characteristics of energy in each frequency band under different operation states, the specific characteristic frequency cannot be reflected. In order to reveal the characteristics of the signal in frequency domain, this paper introduces the power spectrum entropy.

Assuming that the discrete Fourier transform of signal is $X(\omega)$, then the calculation formula for power spectrum is $S(\omega)=(1 / 2 \pi n)|X(\omega)|^{2}$. According to the energy conservation law, $S=\left\{S_{1}, S_{2}, \ldots, S_{n}\right\}$ can be regarded as a division of the signal $X(i)$. Therefore, the power spectrum entropy can be calculated as follows:

(1) The collected acoustic impulse response signal is decomposed with LMD method. Then, compute their power spectrum.

(2) The power spectrum of each PF component is normalized:

$$
\begin{aligned}
P\left(x_{i}\right) & =\frac{S_{i}}{S}, \\
S & =\sum_{i=1}^{n} S_{i} .
\end{aligned}
$$

(3) Calculate the power spectrum entropy:

$$
F(x)=-\sum_{i=1}^{n} P\left(x_{i}\right) \log P\left(x_{i}\right) .
$$

3.4. Extraction of Hilbert Spectrum Entropy Based on LMD. Hilbert spectrum indicates that the amplitude of the signal varies with frequency and time over the entire frequency band. The uniformity of energy distribution in timefrequency directly reflects the difference of signal under different blocking states. Firstly, PF components are preprocessed by Hilbert transform:

$$
H\left[c_{j}(t)\right]=\frac{1}{\pi} \int_{-\infty}^{+\infty} \frac{x(\tau)}{t-\tau} d \tau .
$$

The analytic structure of $x(t)$ is as follows:

$$
x(t)=\operatorname{Re} \sum_{j=1}^{n} a_{i}(t) e^{i \int \omega_{j}(t) d t},
$$

where Re represents the real part and $a_{i}(t)$ represents the instantaneous amplitude.

$$
a_{i}(t)=\sqrt{c_{j}^{2}(t)+H^{2}\left[c_{j}(t)\right]}
$$

Instantaneous frequency is as follows:

$$
f_{i}(t)=\frac{\omega_{j}(t)}{2 \pi} .
$$



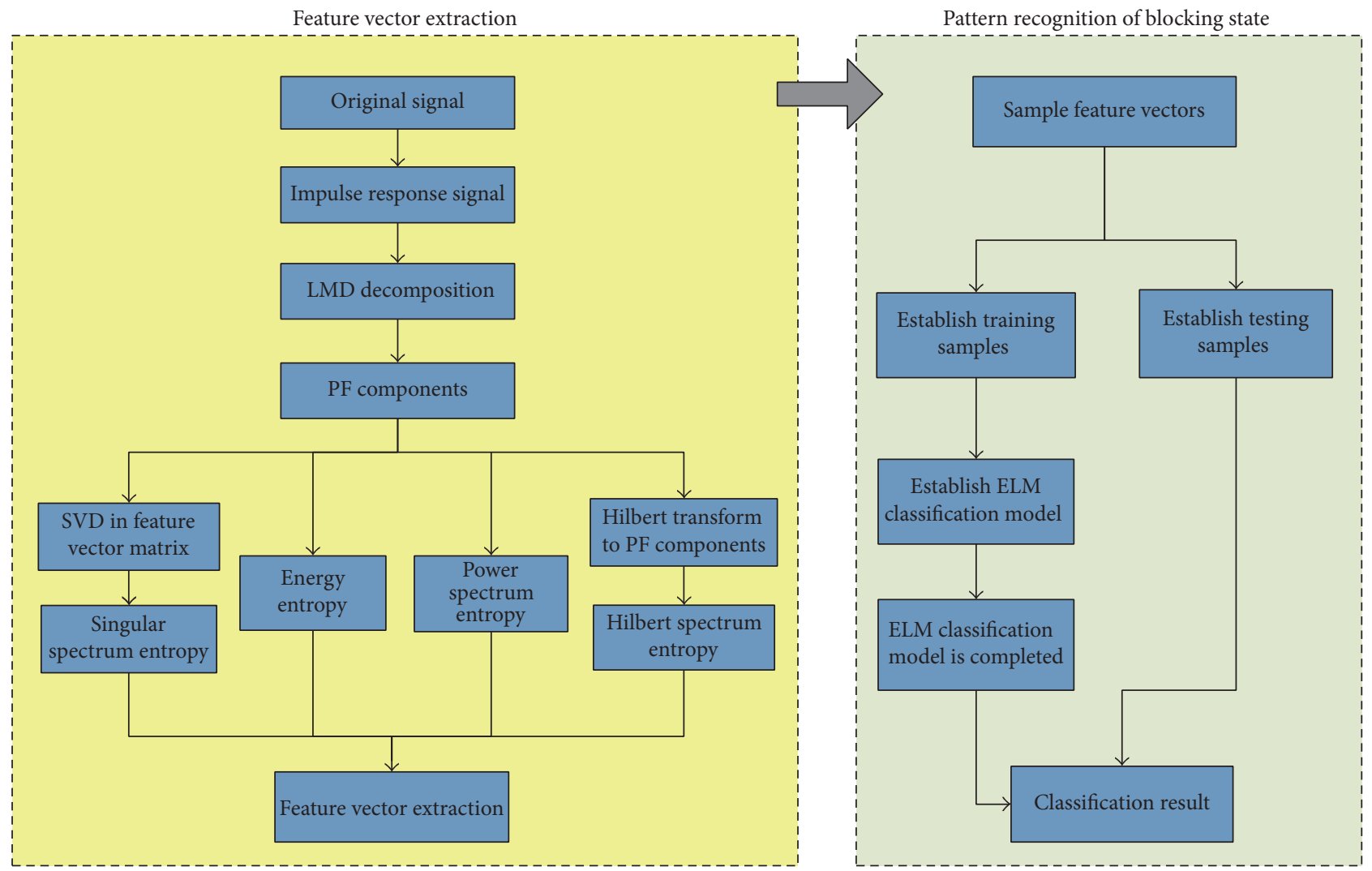

FIGURE 7: Pattern recognition of blocking state in pipeline based on LMD information entropy and ELM.

It can be seen that $x(t)$ is a function of time $t$ and frequency $\omega_{j}(t)$. Furthermore, Hilbert time-frequency spectrum can be expressed as follows:

$$
H(\omega, t)=\operatorname{Re} \sum_{j=1}^{n} b_{i} a_{i}(t) e^{i \int \omega_{j}(t) d t} .
$$

So the calculation step of the Hilbert spectrum entropy is as follows:

(1) The collected acoustic impulse response signal is decomposed with LMD method to obtain a series of PF components. Then, the Hilbert transform is performed and the Hilbert spectrum can be obtained.

(2) The time-frequency plane of Hilbert spectrum is divided into $n$ equal time-frequency blocks. The energy of each block is $H_{i}(i=1,2, \ldots, n)$, and the total energy is $A$. Then, they are normalized as follows:

$$
\begin{aligned}
P\left(x_{i}\right) & =\frac{H_{i}}{A}, \\
A & =\sum_{i=1}^{n} H_{i} .
\end{aligned}
$$

(3) Calculate the Hilbert spectrum entropy:

$$
L(x)=-\sum_{i=1}^{n} P\left(x_{i}\right) \log P\left(x_{i}\right) .
$$

\section{Pattern Recognition Model Based on LMD Information Entropy and ELM}

The specific step based on the pattern recognition of LMD information entropy and ELM is as follows:

(1) The data from health and different degrees of blockage are collected according to a certain sampling frequency acquired by acoustic sensors. Then, calculate the corresponding impulse response by using convolution theorem and the results are used as the experimental data.

(2) In order to get a number of groups of PF components, the acoustic impulse response signals under different conditions are decomposed with LMD method. Because each PF component contains different frequency components (blocking information), the appropriate energy entropy, singular spectrum entropy, power spectrum entropy, and Hilbert spectrum entropy are extracted as the feature vectors.

(3) Build the feature vector of matrix $T_{k}=\left[\sigma_{k 1}, \sigma_{k 2}, \ldots\right.$, $\left.\sigma_{k n}\right]$.

(4) A part of the feature vectors are used to train ELM classifier, and then the rest of the test data is input to ELM for recognizing the blocking state. The whole process of the algorithm is shown in Figure 7 [31]. 


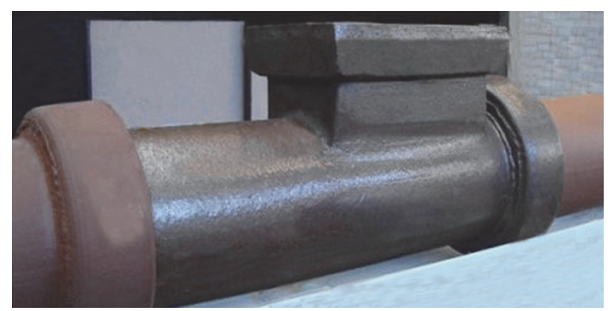

FIgURE 8: Pipeline of $150 \mathrm{~mm}$.

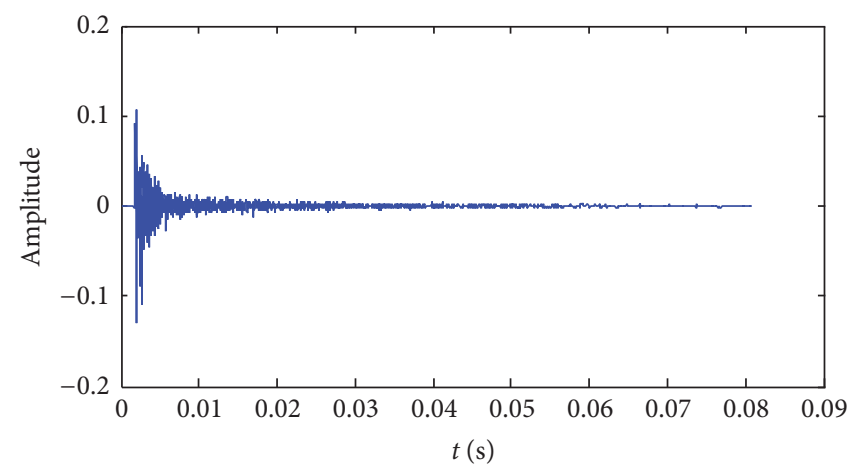

(a) Health

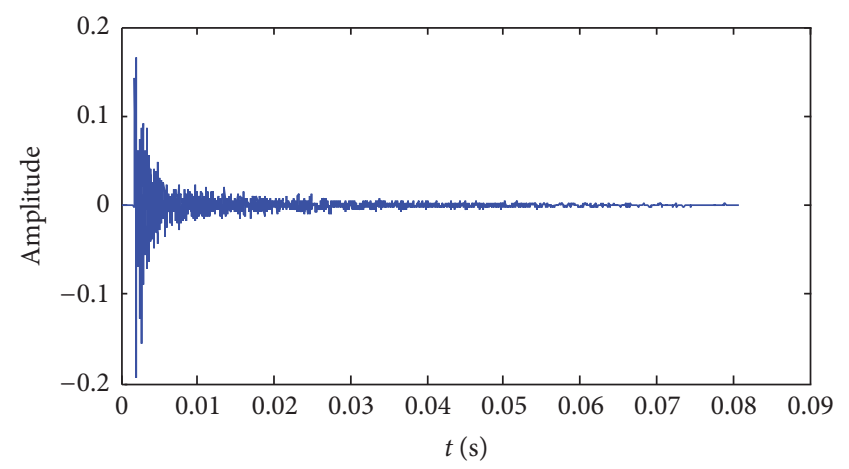

(c) Medium blockage

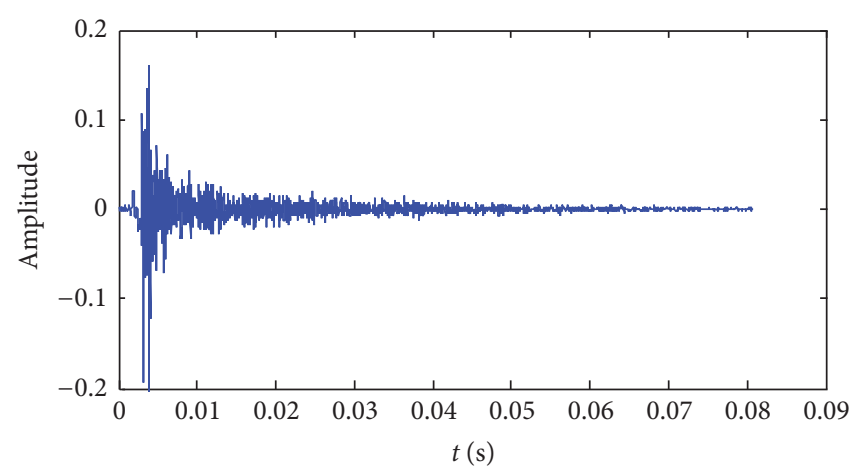

(b) Slight blockage

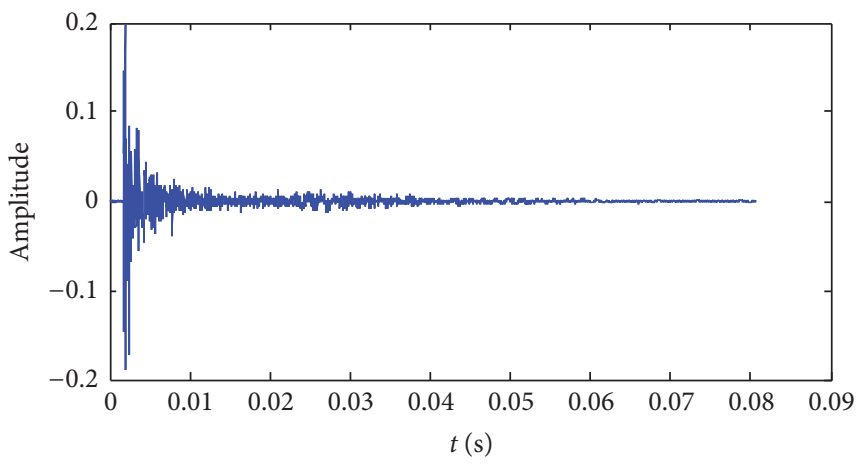

(d) Serious blockage

FIGURE 9: Impulse response signal of pipeline in four kinds of blocking state.

\section{Case Analysis}

5.1. Experiment Data Sources. In order to further verify the effectiveness of the method proposed in this paper, the test used a pipeline of $14.2 \mathrm{~m}$, with internal diameter of $150 \mathrm{~mm}$. The experimental facility used in this work consists of microphone, loudspeaker (Visaton Type K50WP), power amplifier (B\&K Type 2708), and computer. The experimental principle is given in Section 2.2. The computer is used to control the sound card which generated a 10-second sinusoidal sweep. The sound card of computer acts as a signal transmission and acquisition device. At the same time, the power amplifier is used to drive the loudspeaker. When acoustic wave is encountered by the obstacles, part of the acoustic wave can be reflected back. Then, the acoustic signals were recorded on the microphone. The received signals were then deconvolved so that the acoustic impulse response of the pipeline can be obtained. The sampling frequency of the signal is $44100 \mathrm{HZ}$.
As shown in Figure 8 [32], in order to study the condition of blockage, a lateral connection is installed in the middle of the pipeline through which different types of blockages can be deposited at the pipeline.

In these tests, the blockages of different sizes, which occupy $3.6 \%, 14.2 \%$, and $27.8 \%$ of the cross-sectional area of pipeline, are placed in the pipeline respectively. In this experiment, the blockage that occupies less than $10 \%$ of the cross-sectional area of pipeline is defined as slight blockage that occupies 10 20\% of the cross-sectional area of pipeline is defined as medium blockage and that occupies more than $20 \%$ of the cross-sectional area of pipeline is defined as serious blockage. The collected data of four kinds of state which include health, slight blockage, medium blockage, and serious blockage have 40 groups, respectively. Time domain diagram of above four kinds of impulse response signal are shown in Figure 9. Among them, the labels (a), (b), (c), and (d) represent the impulse response signal of pipeline 

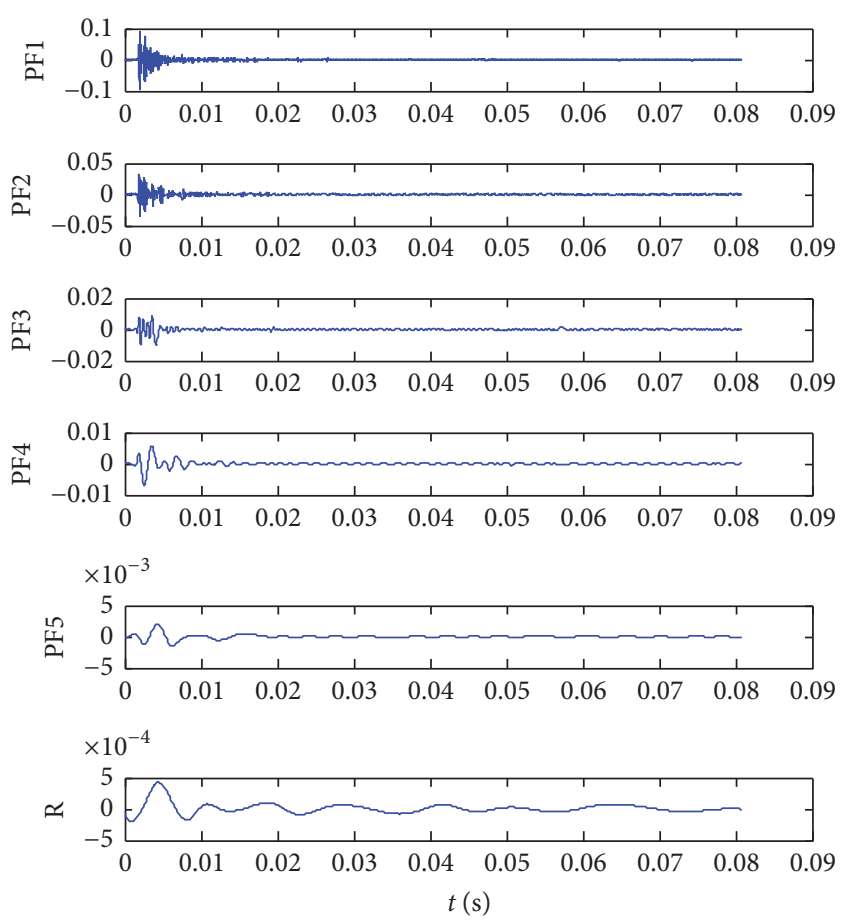

(a)
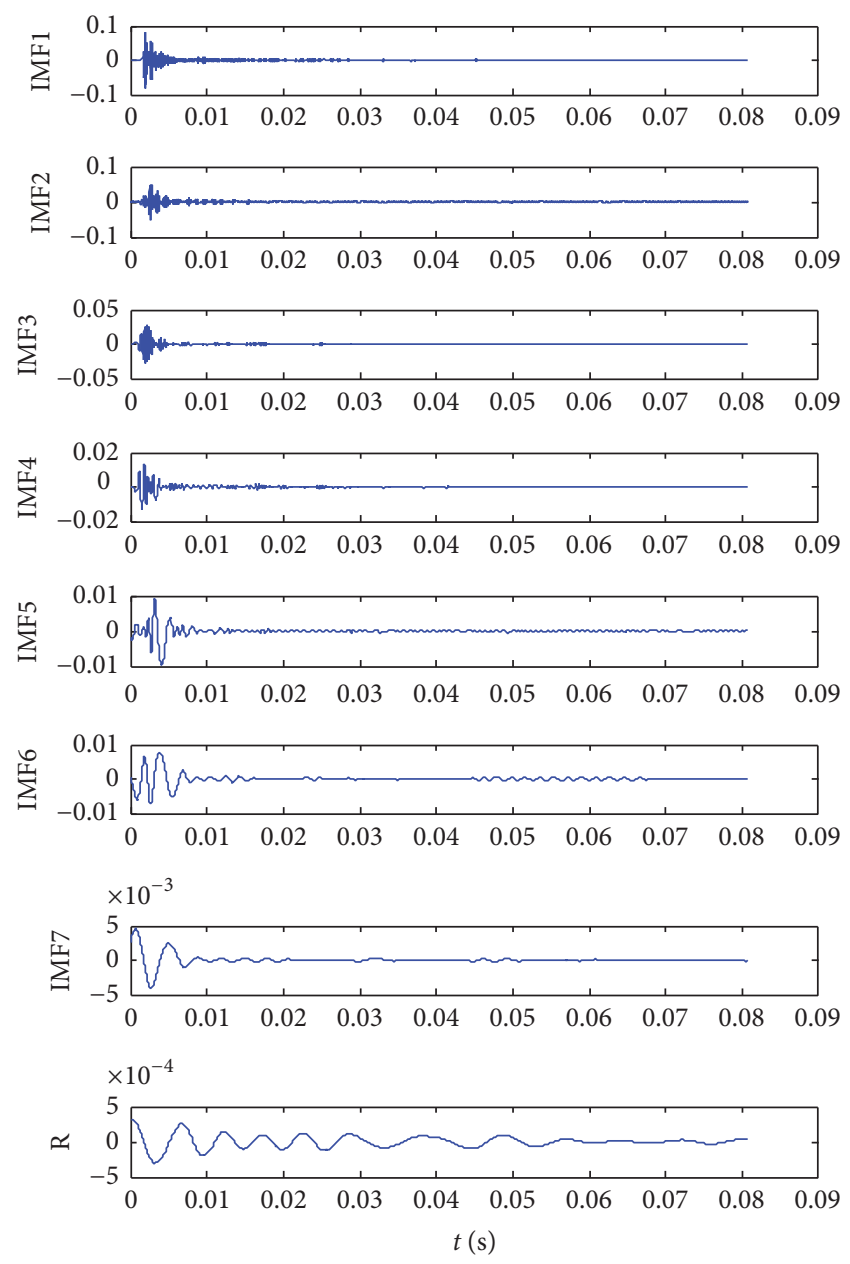

(b)

Figure 10: Results of LMD (a) and EMD (b) decomposition of health.

in health, slight blockage, medium blockage, and serious blockage, respectively. It can be seen from the Figure 9 that the four states of the signal are very similar.

\subsection{Analysis of Feature Extraction Based on LMD Information} Entropy. In order to obtain the corresponding state of feature vector, the signals of health and varying degrees of blockage were decomposed into a series of PF components by LMD, respectively. As shown in Figures 10(a), 11(a), 12(a), and 13(a), the state information of the signal is concentrated in the first 5 PF components. For comparison purposes, the signals were also decomposed by EMD, respectively. Due to space limitations, this paper only listed the first 8 IMF components. As shown in Figures 10(b), 11(b), 12(b), and 13(b), there are too many redundant components.

For ease of comparison studies, the partial feature vectors and the average value of the singular spectrum entropy, energy entropy, power spectrum entropy, and Hilbert spectrum entropy obtained by LMD and EMD methods are given in Table 1 and Figure 14. Among them, the labels A, B, C, and $\mathrm{D}$ represent the singular spectrum entropy, energy entropy, power spectrum entropy, and Hilbert spectrum entropy, respectively. As shown in Table 1 and Figure 14, it can be found by comparison that the same kinds of characteristics of information entropy values which are represented in different blocking states show certain difference with LMD information entropy method, and the distinguish degree is high. Meanwhile, with the deepening of the degree of blockage, the average value of the four types of information entropy shows a gradually decreasing trend generally, as shown in Figure 14(a). When the pipeline is blocked, the distribution of the energy, frequency, and other pieces of information in impulse response signal can be more focused on the part of blockage. However, in the health state, the distribution is more dispersed. Therefore, the uncertainty degree of impulse response signal which is in health state can be greater than the blocking state. Also, it can be seen that the average value of singular spectrum entropy (a) and energy entropy (b) are not completely decreased with the increase of the degree of blockage from Figure 14(a). This is mainly because the acoustic impulse response signal which reflects the operating state of the pipeline is a complex nonstationary and nonlinear signal. Although time domain and timefrequency domain features can provide some information to 

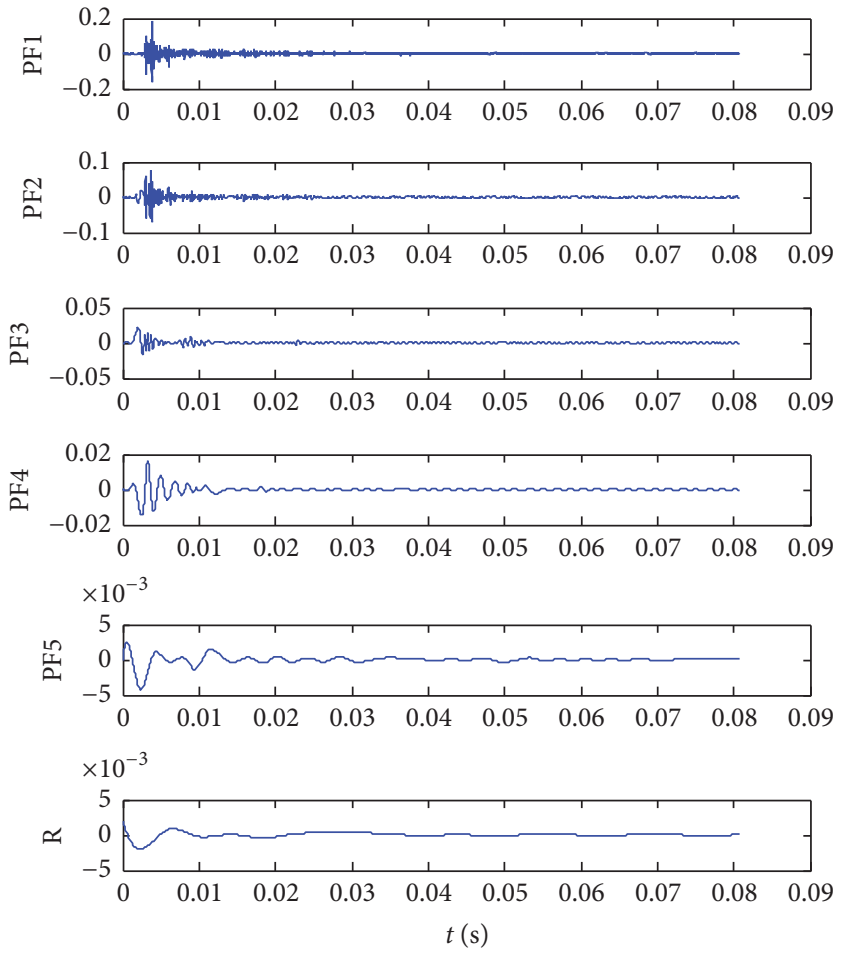

(a)

FIgURE 11: Results of LMD (a) and EMD (b) decomposition of slight blockage.

reflect the state of operation, it is not enough for pattern recognition. Through the combination of the characteristics in time domain, frequency domain, and time-frequency domain, it can reflect the state information of blockage with more accuracy.

In addition, although the four kinds of feature vectors extracted by EMD information entropy method show certain distinction degree in expressing different types of blockage, some of the information entropy values under different blocking states have a certain degree of cross-aliasing when comparing the average value of the four types of information entropy, which made the discrimination not obvious. As shown in Figure 14(b), with the deepening of the degree of blockage, the average value of the four types of information entropy does not clearly show gradually decreasing trend.

Because LMD method is better than EMD method in iterative efficiency and suppression of the endpoint effect, the instantaneous frequency components separated by LMD are more realistic, and the result have a few false frequency components. LMD method can not only reduce the degree of modal mixture caused by EMD decomposition but also obtain some PF components which contain a wealth of
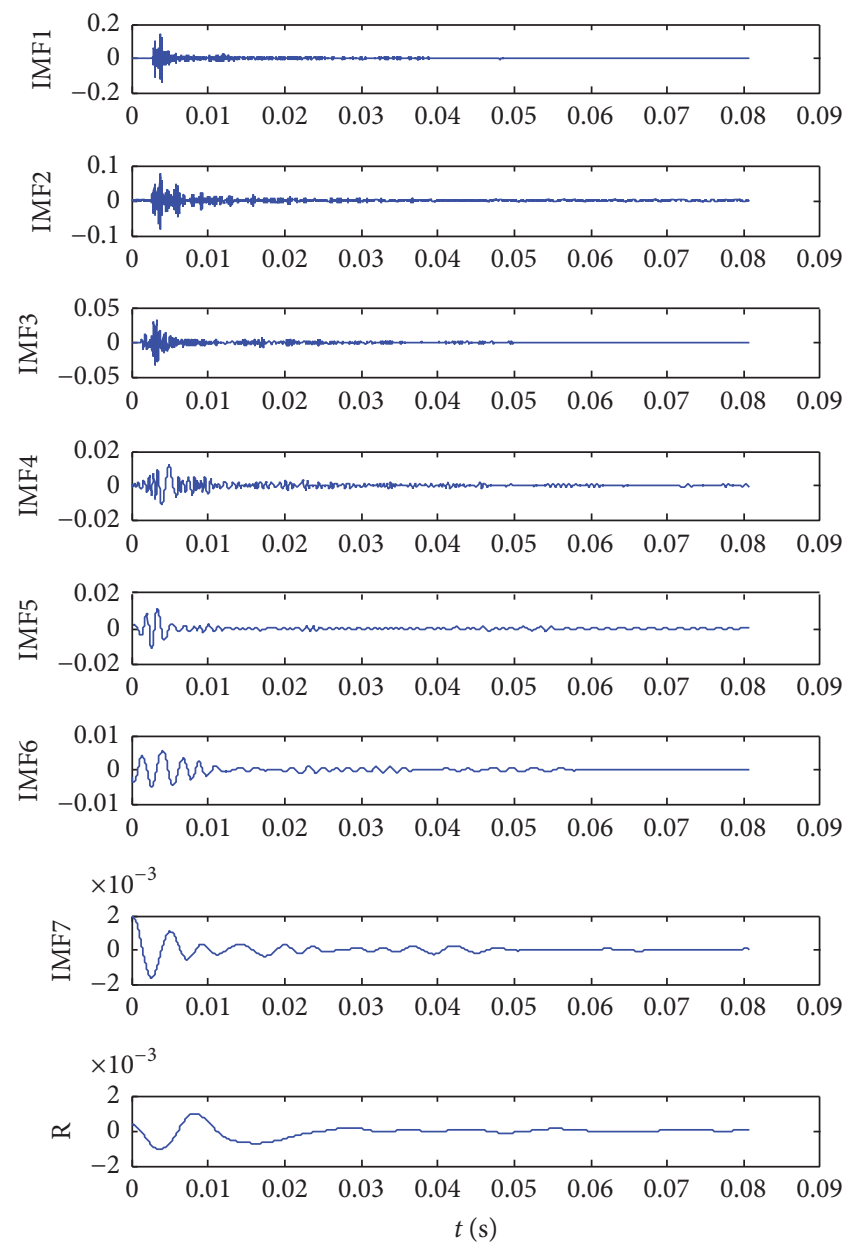

(b)

information. Meanwhile, it can be seen that the discrimination of information entropy decomposed by LMD is better than EMD from the experimental results, and it is more accurate to describe the uncertainty degree of the system.

5.3. Pipeline Blocking State Classification Based on ELM. In this paper, we selected a total of 160 groups of feature vectors as the research object, which have 40 groups for each kind of blocking state that include health, slight blockage, medium blockage, and serious blockage. The first 28 groups of feature vectors for each kind of blocking state were used as the training samples, and the remaining 12 groups were used as the testing samples. After the training of ELM classifier, the testing samples were input into the classification model for recognizing the blocking state. Among them, the number of hidden layer nodes in ELM is set to 18, and the activation function of the hidden layer is the common type of sigmoid logarithm. In order to verify the validity of the proposed method, the results of feature extraction based on LMD information entropy and EMD information entropy were all input into ELM to carry out the classification, and the classification results are shown in Table 2. 


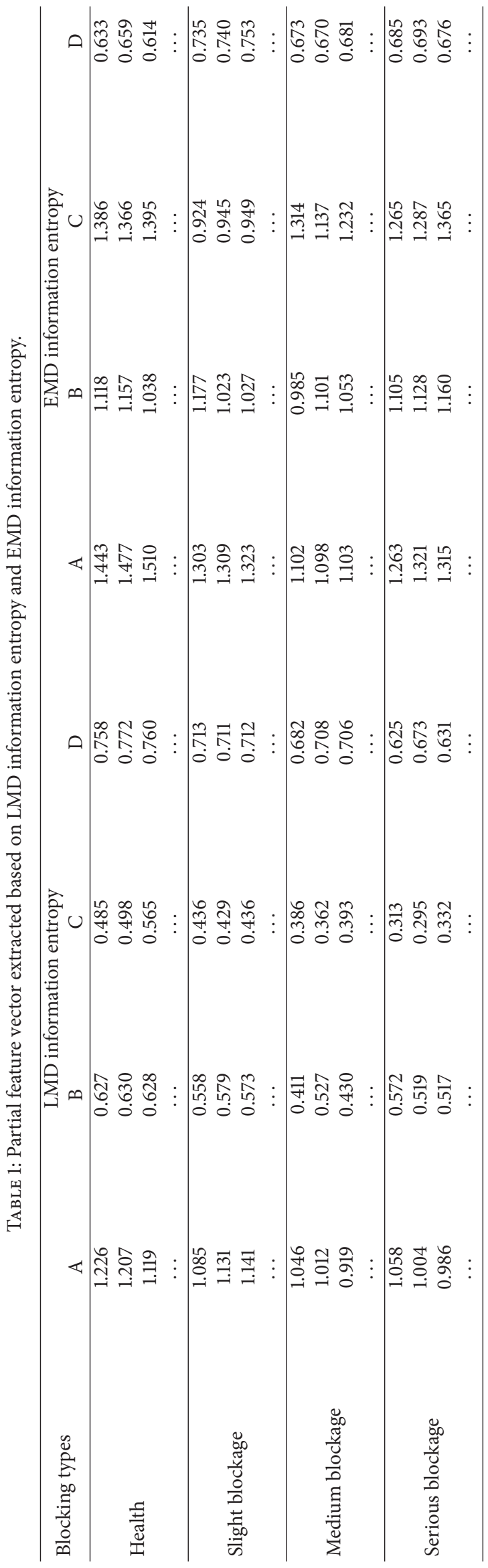



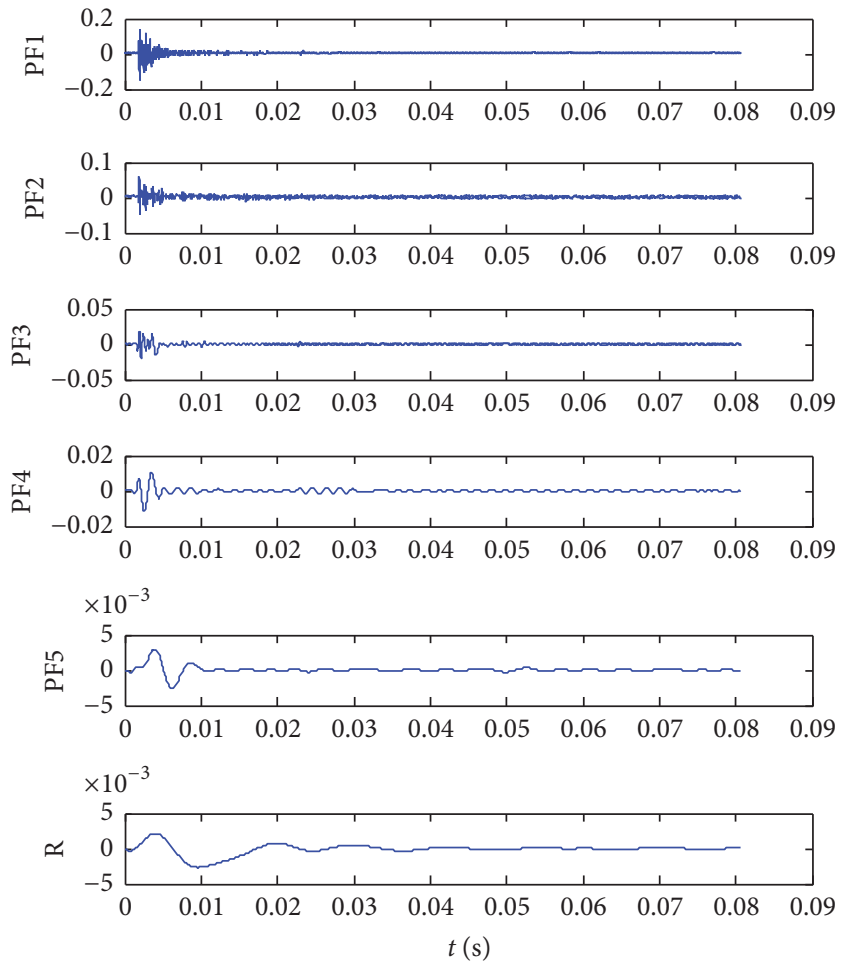

(a)
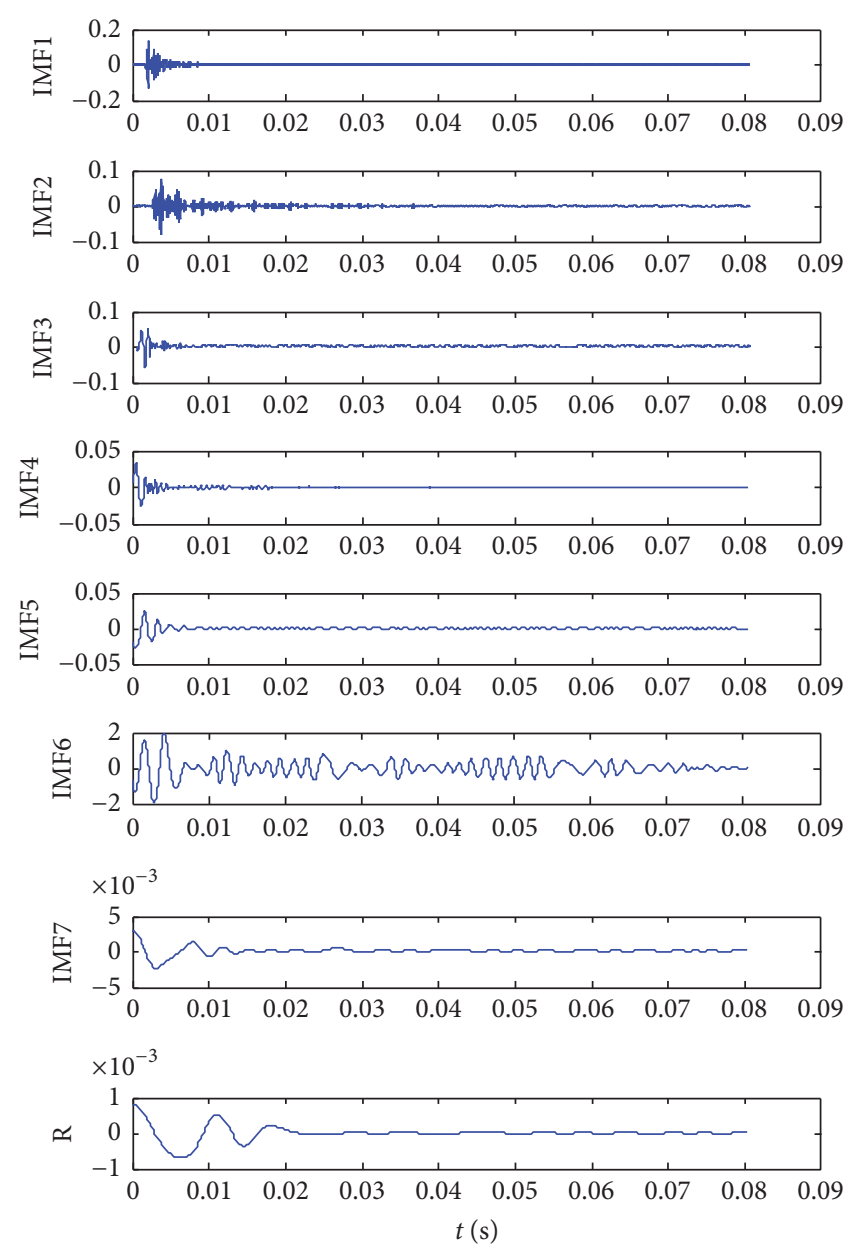

(b)

FIgURE 12: Results of LMD (a) and EMD (b) decomposition of medium blockage.

TABLE 2: Test results of two methods.

\begin{tabular}{|c|c|c|}
\hline \multirow[b]{2}{*}{ State } & \multicolumn{2}{|c|}{ Classification accuracy } \\
\hline & $\begin{array}{c}\text { LMD information } \\
\text { entropy }\end{array}$ & $\begin{array}{c}\text { EMD information } \\
\text { entropy }\end{array}$ \\
\hline Health & 1 & 0.9167 \\
\hline Slight blockage & 0.8333 & 0.8333 \\
\hline Medium blockage & 0.8333 & 0.75 \\
\hline Serious blockage & 0.9167 & 0.75 \\
\hline Average value & 0.8958 & 0.8125 \\
\hline
\end{tabular}

As shown in Table 2, there are five errors which are incorrectly recognized in the testing samples by using LMD information entropy method, and the average recognition rate is $89.58 \%$. However, the recognition results based on EMD information entropy method are lower, and the average recognition rate is $81.25 \%$. Therefore, the pattern recognition method based on LMD Information entropy can gain good recognition effect.

In order to further prove the effectiveness of the proposed method in this paper, the test collected extra 10 times experimental data in the same way and extracted 40 groups of feature vectors for each kind of blocking state in each time. At the same time, the first 28 groups of feature vectors for each kind of blocking state were used as training samples, and the remaining 12 groups were used as testing samples. Then, a total of 10 times classification test was made between ELM, BP, and SVM in terms of running time and classification accuracy. In BP model, the activation function is the commonly used sigmoid function, and the number of hidden layer nodes is 4. In SVM model, the test selected RBF as the kernel. Meanwhile, the penalty parameter is set to 500, and the bandwidth of the RBF kernel is set to 1.7. The test started with Matlab R 2009. The detailed results are shown in Table 3 and Figure 15. It can be seen from Figure 15 that the time spend on BP classifier is significantly higher than the other two methods, and the classification accuracy is relatively low. Meanwhile, although the classification accuracy of SVM is slightly lower than that of ELM, the ELM has a great advantage in terms of running time. Compared with ELM, SVM has two parameters which need to be man-made. However, ELM only needs to set the number of hidden layer neurons. Although there are 

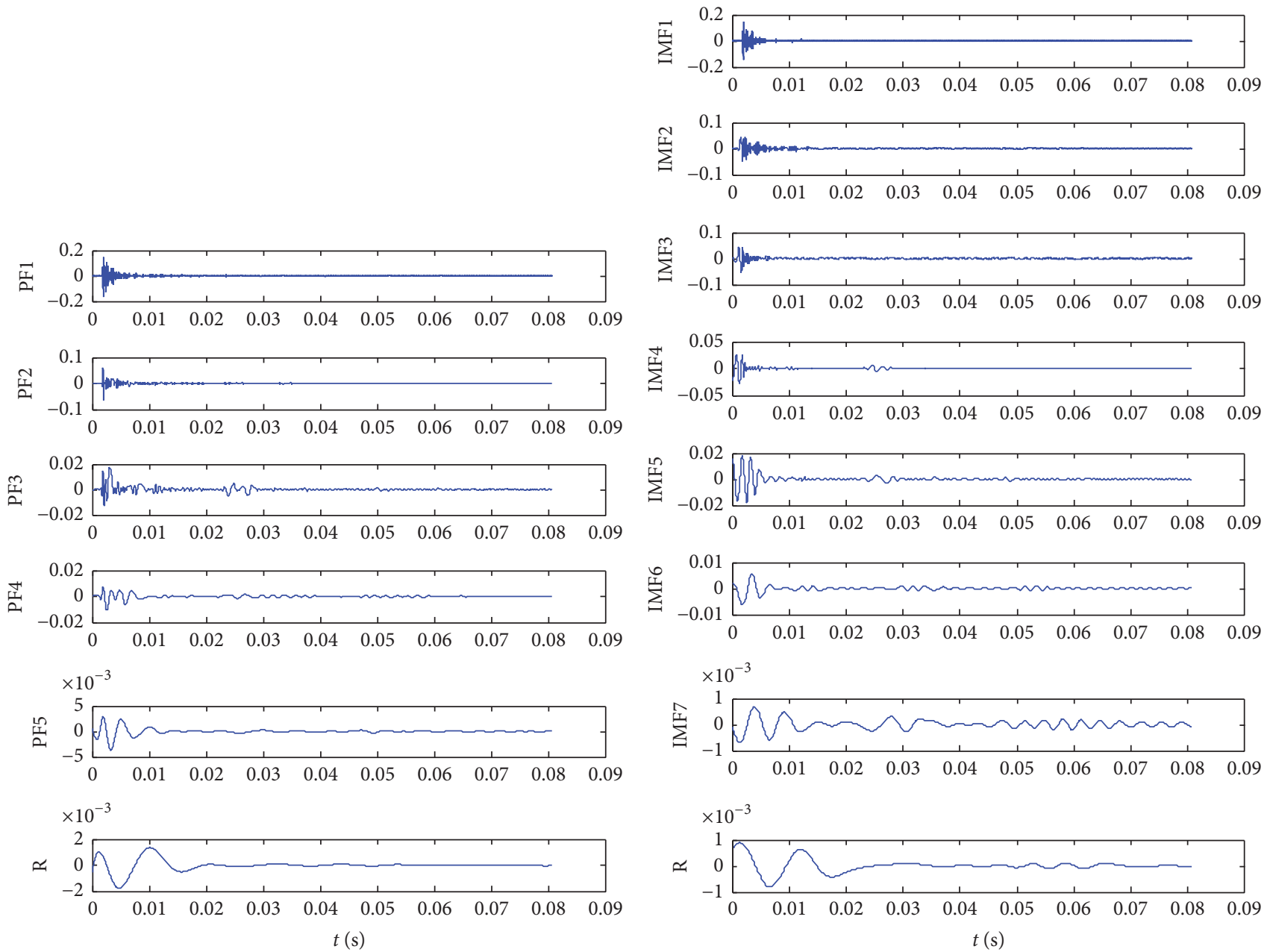

(a)

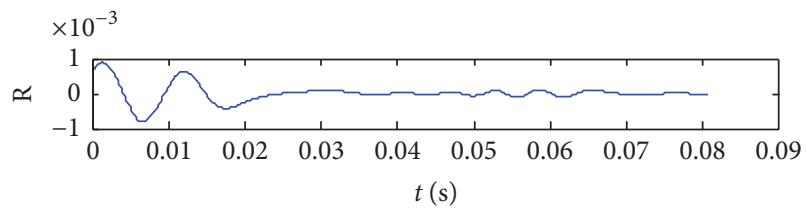

(b)

FIGURE 13: Results of LMD (a) and EMD (b) decomposition of serious blockage.

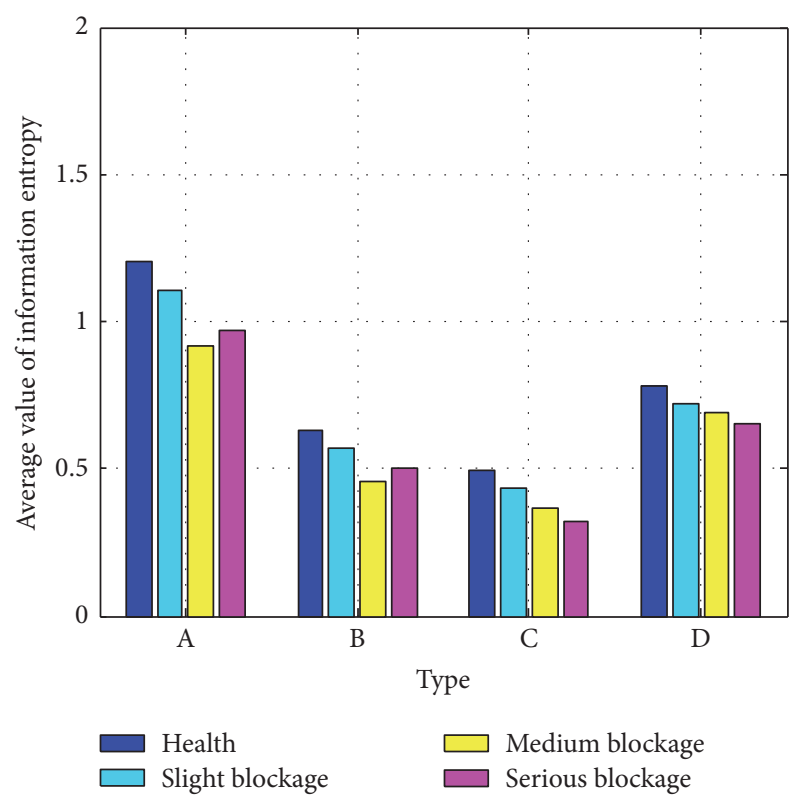

(a)

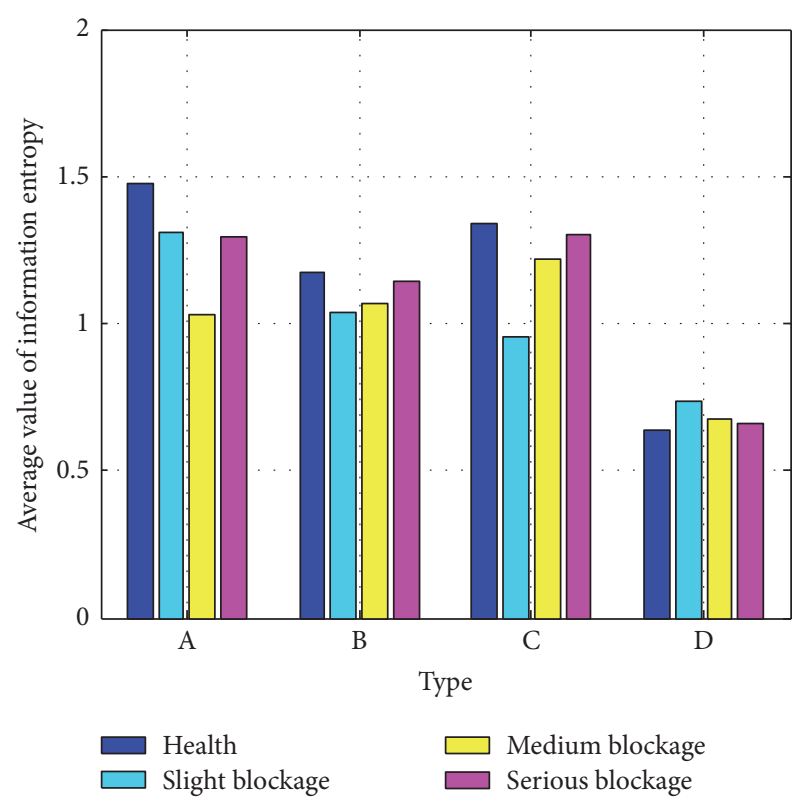

(b)

FIGURE 14: The average value of feature vectors extracted based on LMD information entropy (a) and EMD information entropy (b). 

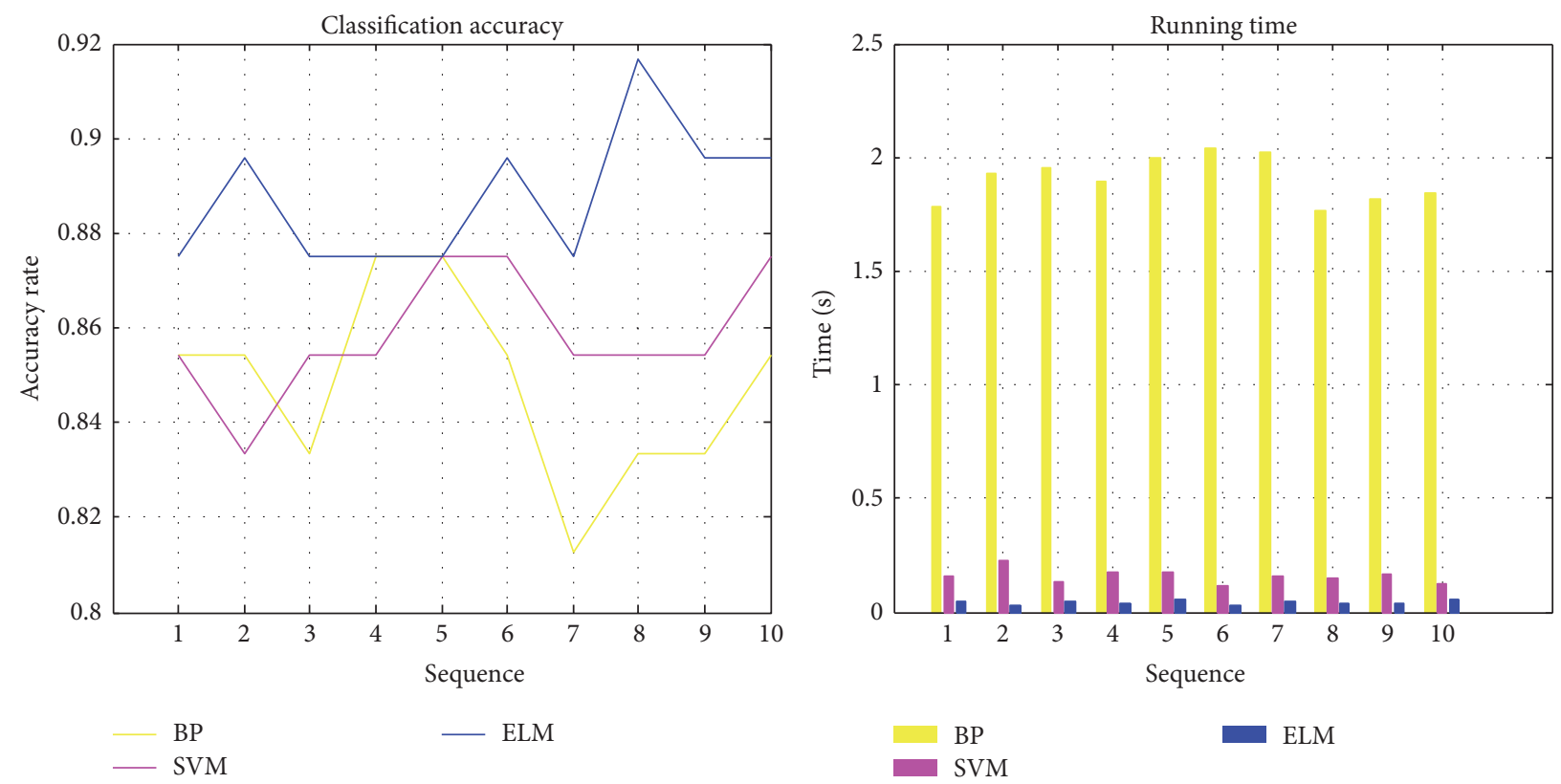

FIgURE 15: Classification results of ELM, SVM, and BP.

TABLE 3: Classification results of ELM, SVM, and BP.

\begin{tabular}{|c|c|c|c|c|c|c|}
\hline \multirow{2}{*}{ Sequence } & \multicolumn{3}{|c|}{ Running time/s } & \multicolumn{3}{|c|}{ Classification accuracy } \\
\hline & $\mathrm{BP}$ & SVM & ELM & $\mathrm{BP}$ & SVM & ELM \\
\hline 1 & 1.7854 & 0.1590 & 0.0433 & 0.8542 & 0.8542 & 0.8750 \\
\hline 2 & 1.9260 & 0.2243 & 0.0328 & 0.8542 & 0.8333 & 0.8958 \\
\hline 3 & 1.9569 & 0.1365 & 0.0501 & 0.8333 & 0.8542 & 0.8750 \\
\hline 4 & 1.8934 & 0.1733 & 0.0423 & 0.8750 & 0.8542 & 0.8750 \\
\hline 5 & 1.9987 & 0.1764 & 0.0536 & 0.8750 & 0.8750 & 0.8750 \\
\hline 6 & 2.0363 & 0.1197 & 0.0312 & 0.8542 & 0.8750 & 0.8958 \\
\hline 7 & 2.0258 & 0.1611 & 0.0462 & 0.8125 & 0.8542 & 0.8750 \\
\hline 8 & 1.7672 & 0.1461 & 0.0397 & 0.8333 & 0.8542 & 0.9167 \\
\hline 9 & 1.8137 & 0.1713 & 0.0362 & 0.8333 & 0.8542 & 0.8958 \\
\hline 10 & 1.8392 & 0.1265 & 0.0575 & 0.8542 & 0.8750 & 0.8958 \\
\hline
\end{tabular}

many existing parameters optimization algorithm, it is still a difficult problem to select a suitable algorithm for the specific problem. In addition, it can be seen from the results that it can gain good recognition effect by using above three models. This is because the extracted feature vectors of pipeline which is in different blocking state have a good discrimination, so as to prove the effectiveness of the proposed method in this paper.

\section{Conclusions}

Since the characteristic of the acoustic impulse response signal in pipeline blockage is nonstationary and nonlinear, this paper combined LMD, information entropy, and ELM algorithm for diagnosis and analysis of the different degrees of blockage. The conclusions are obtained as follows:

(1) The experimental results show that the information entropy combined with time domain, frequency domain, and time-frequency domain information can be well used to measure the operation state of pipeline, which means that it can better express the state information of the signal.

(2) Because LMD method is better than EMD method in iterative efficiency and suppression of the endpoint effect, the discrimination of extracted information entropy has better results than EMD method, according to the tests performed in this paper. Moreover, the experimental results show that the average recognition rate of the model based on LMD information entropy and ELM is $89.58 \%$, and the average recognition rate based on EMD information entropy and ELM model is only $81.25 \%$, which proved the validity of the proposed method.

(3) The results of comparative experiment also further proved the effectiveness of the proposed method. Compared with BP and SVM classifiers, ELM has 
achieved good results in terms of accuracy and time consuming, which highlights the advantages of ELM in pattern recognition. It provides a feasible way for the pattern recognition method of blocking state in pipeline.

\section{Competing Interests}

The authors declare that there are no competing interests regarding the publication of this paper.

\section{Acknowledgments}

This work was supported by the National Natural Science Foundation of China (61563024, 51169007, and 61663017) and the Science \& Research Program of Yunnan Province (2015ZC005).

\section{References}

[1] S. Datta and S. Sarkar, "A review on different pipeline fault detection methods," Journal of Loss Prevention in the Process Industries, vol. 41, pp. 97-106, 2016.

[2] D. B. Sharp and D. M. Campbell, "Leak detection in pipes using acoustic pulse reflectometry," Acustica, vol. 83, no. 3, pp. 560566, 1997.

[3] U. Yunus, M. Tsunasaki, Y. He, M. Kominami, and K. Yamashita, "Detection of leak location in a pipeline by acoustic signal," IEICE Transactions on Fundamentals of Electronics, Communications \& Computer Sciences, vol. 91, no. 8, pp. 2053-2061, 2008.

[4] L. L. Silva, P. C. C. Monteiro, J. L. A. Vidal, and T. A. Netto, "Acoustic reflectometry for blockage detection in pipeline," in Proceedings of the ASME 33rd International Conference on Ocean, Offshore and Arctic Engineering (OMAE '14), June 2014.

[5] J. S. Smith, "The local mean decomposition and its application to EEG perception data," Journal of the Royal Society Interface, vol. 2, no. 5, pp. 443-454, 2005.

[6] J.-S. Cheng, K. Zhang, Y. Yang, and D.-J. Yu, "Comparison between the methods of local mean decomposition and empirical mode decomposition," Journal of Vibration and Shock, vol. 28, no. 5, pp. 13-16, 2009 (Chinese).

[7] Y. Yu, YuDejie, and C. Junsheng, "A roller bearing fault diagnosis method based on EMD energy entropy and ANN," Journal of Sound and Vibration, vol. 294, no. 1-2, pp. 269-277, 2006.

[8] R. Yan and R. X. Gao, "Approximate Entropy as a diagnostic tool for machine health monitoring," Mechanical Systems and Signal Processing, vol. 21, no. 2, pp. 824-839, 2007.

[9] E. Cabal-Yepez, R. J. Romero-Troncoso, A. Garcia-Perez, and R. A. Osornio-Rios, "Single-parameter fault identification through information entropy analysis at the startup-transient current in induction motors," Electric Power Systems Research, vol. 89, pp. 64-69, 2012.

[10] J. Lai, J. Qiu, Z. Feng, J. Chen, and H. Fan, "Prediction of soil deformation in tunnelling using artificial neural networks," Computational Intelligence and Neuroscience, vol. 2016, Article ID 6708183, 16 pages, 2016.

[11] R. B. Dhumale and S. D. Lokhande, "Neural network fault diagnosis of voltage source inverter under variable load conditions at different frequencies," Measurement, vol. 91, pp. 565-575, 2016.
[12] J. Ben Ali, N. Fnaiech, L. Saidi, B. Chebel-Morello, and F. Fnaiech, "Application of empirical mode decomposition and artificial neural network for automatic bearing fault diagnosis based on vibration signals," Applied Acoustics, vol. 89, pp. 16-27, 2015.

[13] G.-B. Huang, Q.-Y. Zhu, and C.-K. Siew, "Extreme learning machine: theory and applications," Neurocomputing, vol. 70, no. 1-3, pp. 489-501, 2006.

[14] Y. Tian, J. Ma, C. Lu, and Z. Wang, "Rolling bearing fault diagnosis under variable conditions using LMD-SVD and extreme learning machine," Mechanism and Machine Theory, vol. 90, pp. 175-186, 2015.

[15] S. Scardapane, D. Comminiello, M. Scarpiniti, and A. Uncini, "Music classification using extreme learning machines," in Proceedings of the 8th International Symposium on Image and Signal Processing and Analysis (ISPA '13), pp. 377-381, September 2013.

[16] D. S. Karpagachelvi, "Classification of ECG signals using particle swarm optimization and extreme learning, machine," International Journal of Engineering Sciences \& Research Technology, vol. 3, no. 7, pp. 95-102, 2014.

[17] L. E. Kinsler and A. R. Frey, "Fundamental of Acoustics," 1982.

[18] A. P. Dowling, J. E. F. Williams, and W. M. Wright, Sound and Sources of Sound, E. Horwood, 1983.

[19] A. D. Pierce, "Acoustics: an introduction to its physical principles and applications," Physics Today, vol. 34, no. 12, pp. 18261827, 1981.

[20] T. Liu, Study on the signal processing technique for pipeline inspection system [Ph.D. dissertations], Tianjin University, 2007 (Chinese).

[21] J. W. Gooch, Snell's Law, Springer, New York, NY, USA, 2011.

[22] D. W. Steeples and R. D. Miller, "Seismic reflection methods applied to engineering, environmental, and ground-water problems," in Proceedings of the 1st EEGS Symposium on the Application of Geophysics to Engineering and Environmental Problems, Investigations in Geophysics, Golden, Colo, USA, 1988.

[23] G.-B. Stan, J.-J. Embrechts, and D. Archambeau, "Comparison of different impulse response measurement techniques," AES: Journal of the Audio Engineering Society, vol. 50, no. 4, pp. 249262, 2002.

[24] K. A. Papadopoulou, M. N. Shamout, B. Lennox et al., "An evaluation of acoustic reflectometry for leakage and blockage detection," Proceedings of the Institution of Mechanical Engineers, Part C: Journal of Mechanical Engineering Science, vol. 222, no. 6, pp. 959-966, 2008.

[25] M. Holters, T. Corbach, and U. Zölzer, "Impulse response measurement techniques and their applicability in the real world," in Proceedings of the 12th International Conference on Digital Audio Effects (DAFx '09), pp. 108-112, ISPG, Como, Italy, September 2009.

[26] J. Cheng, Y. Yang, and Y. Yang, "A rotating machinery fault diagnosis method based on local mean decomposition," Digital Signal Processing, vol. 22, no. 2, pp. 356-366, 2012.

[27] S. Zhang, P. Li, L. Zhang, H. Li, W. Jiang, and Y. Hu, "Modified S transform and ELM algorithms and their applications in power quality analysis," Neurocomputing, vol. 185, pp. 231-241, 2016.

[28] C. E. Shannon, "A mathematical theory of communication," ACM SIGMOBILE Mobile Computing and Communications Review, vol. 5, no. 1, pp. 3-55, 2001.

[29] L. R. Nemzer, "Shannon information entropy in the canonical genetic code," Journal of Theoretical Biology, vol. 415, pp. 158170, 2017. 
[30] V. C. Klema and A. J. Laub, “The singular value decomposition: its computation and some applications," IEEE Transactions on Automatic Control, vol. 25, no. 2, pp. 164-176, 1980.

[31] L. Zhao, W. Yu, and R. Yan, "Rolling bearing fault diagnosis based on CEEMD and time series modeling," Mathematical Problems in Engineering, vol. 2014, Article ID 101867, 13 pages, 2014.

[32] Z. Feng, Condition classification in underground pipes based on acoustical characteristics [Ph.D. thesis], University of Bradford, Bradford, UK, 2013. 


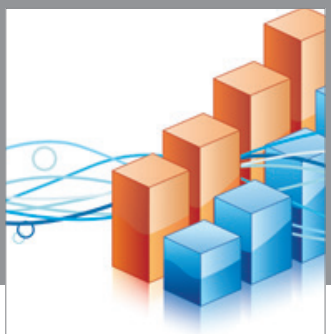

Advances in

Operations Research

vatem alat4

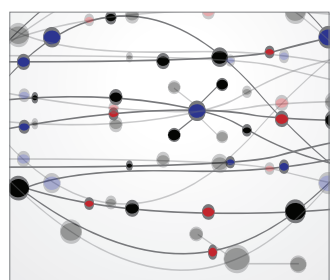

\section{The Scientific} World Journal
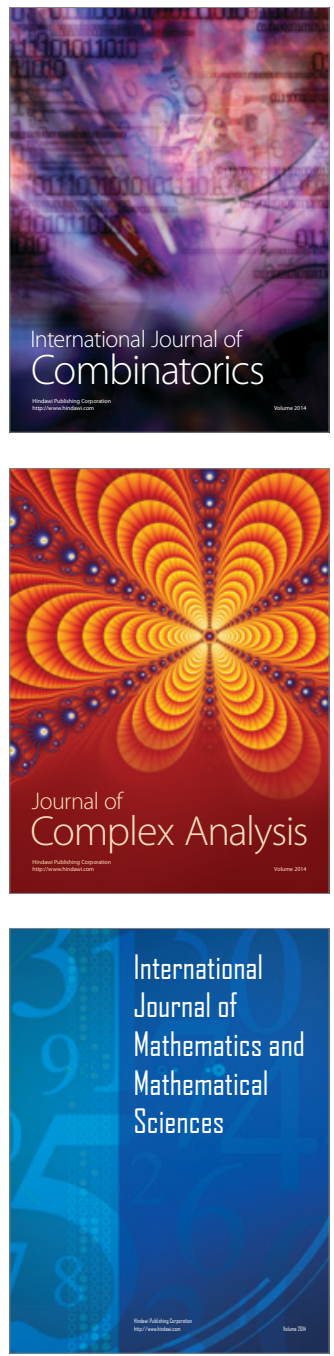
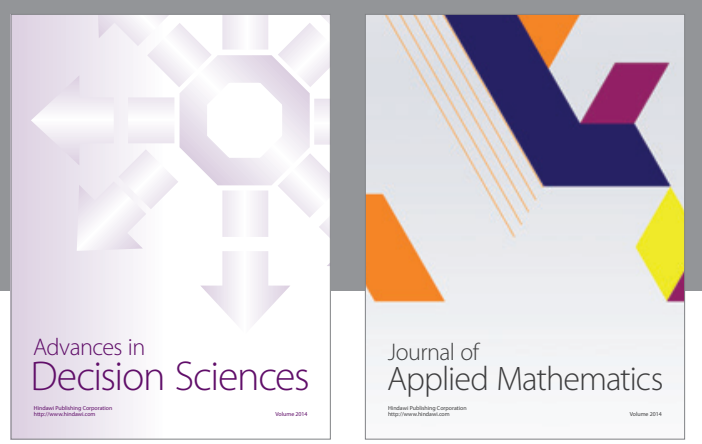

Algebra

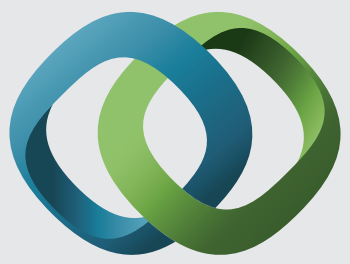

\section{Hindawi}

Submit your manuscripts at

https://www.hindawi.com
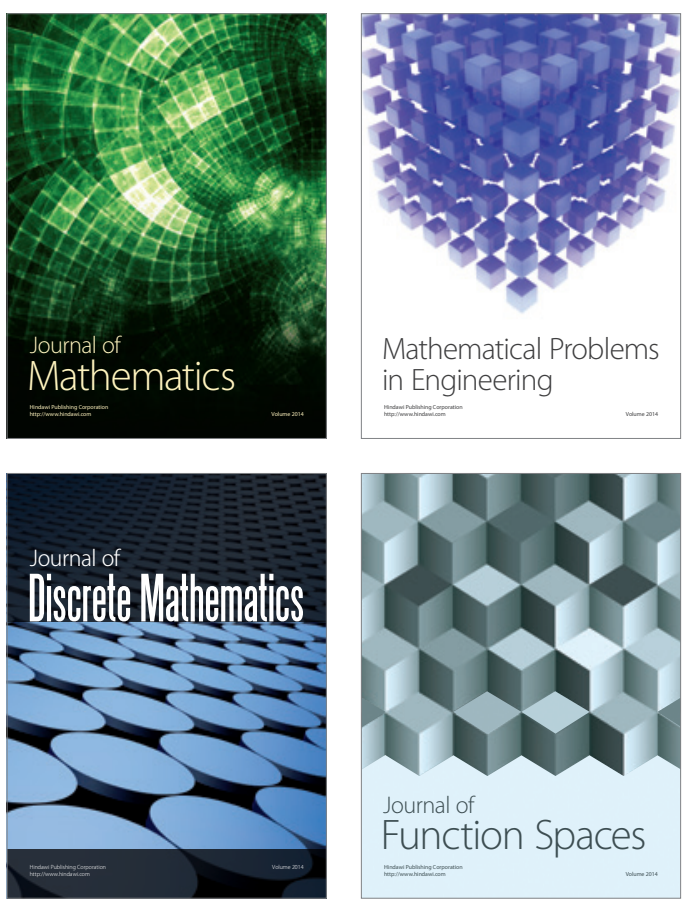

Mathematical Problems in Engineering
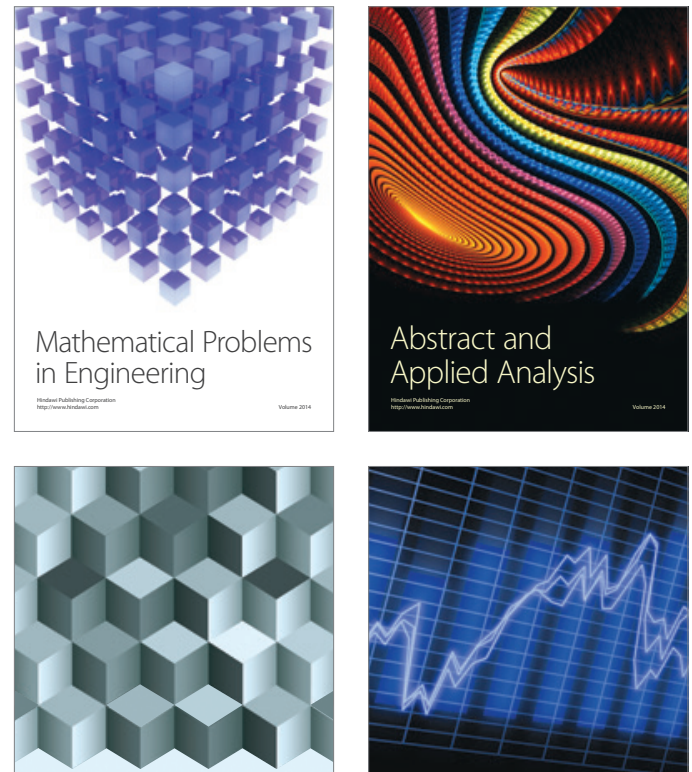

Journal of

Function Spaces

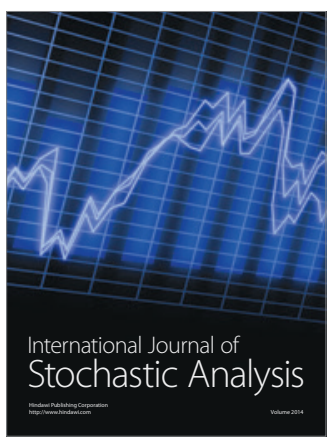

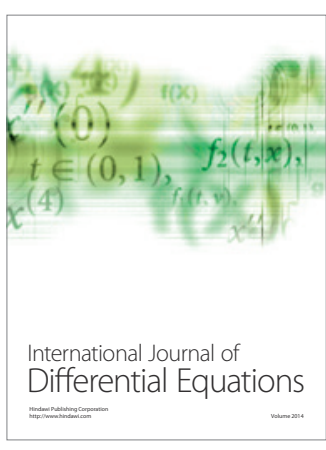
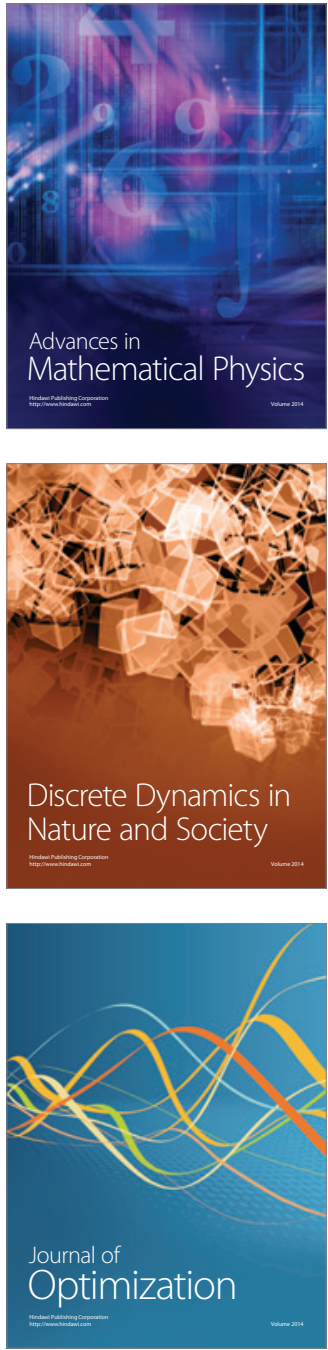